\title{
Pacific
}

Journal of

Mathematics

\section{A REMARK ON EINSTEIN WARPED PRODUCTS}

MiCHELE RIMOLDI 


\title{
A REMARK ON EINSTEIN WARPED PRODUCTS
}

\author{
MiCHELE RiMOLDI
}

\begin{abstract}
We prove triviality results for Einstein warped products with noncompact bases. These extend previous work by Kim and Kim. The proofs, from the viewpoint of quasi-Einstein manifolds introduced by Case, Shu and Wei, rely on maximum principles at infinity and Liouville-type theorems.
\end{abstract}

\section{Introduction}

The main purpose of this note is to prove the following triviality result for Einstein warped products, which extends a theorem by Kim and Kim [2003] to the case of noncompact bases.

Theorem 1. Let $N^{n+m}=M^{n} \times_{u} F^{m}$, with $m>1$, be a complete Einstein warped product with nonpositive scalar curvature ${ }^{N} S \leq 0$, warping function $u(x)=e^{-f(x) / m}$ satisfying $\inf _{M} f=f_{*}>-\infty$ and complete Einstein fibre $F$. Then $N$ is simply a Riemannian product if either of these conditions is satisfied:

(a) The function $f$ has a local minimum.

(b) The base manifold $M$ is complete and noncompact, the warping function satisfies $\int_{M}|f|^{p} e^{-f / m} d \mathrm{vol}<+\infty$, for some $1<p<+\infty$, and $f\left(x_{0}\right) \leq 0$ for some point $x_{0} \in M$.

If $M$ is compact, from (a) we recover the main result in [Kim and Kim 2003].

Our proof of Theorem 1 will rely on the link between Einstein warped product metrics and the quasi-Einstein metrics recently introduced by Case, Shu and Wei [2011]. In the spirit of [Pigola et al. 2011], that is, using methods from stochastic analysis and $L^{p}$-Liouville-type theorems, we shall prove scalar curvature estimates and triviality results for a complete quasi-Einstein manifold that largely extend previous theorems in [Case et al. 2011]. The main theorem will follow immediately.

In a final section, using similar techniques, we extend another triviality result for Einstein warped products obtained in [Case 2010]. A nonexistence result is also discussed.

Keywords: Einstein warped products, quasi-Einstein manifolds, triviality, scalar curvature. 


\section{Quasi-Einstein manifolds}

Consider the weighted manifold $\left(M^{n}, g_{M}, e^{-f} d\right.$ vol), where $M$ is a complete $n$ dimensional Riemannian manifold, $f$ is a smooth real valued function on $M$ and $d$ vol is the Riemannian volume density on $M$. A natural extension of the Ricci tensor to weighted manifolds is the $m$-Bakry-Emery Ricci tensor

$$
\operatorname{Ric}_{f}^{m}=\operatorname{Ric}+\operatorname{Hess}(f)-\frac{1}{m} d f \otimes d f, \quad \text { for } 0<m \leq \infty .
$$

When $f$ is constant this is the usual Ricci tensor, and when $m=\infty$ this is the Ricci Bakry-Emery tensor Ric $_{f}$. We call a metric $m$-quasi-Einstein if the $m$-BakryEmery Ricci tensor satisfies the equation

$$
\operatorname{Ric}_{f}^{m}=\lambda g_{M},
$$

for some $\lambda \in \mathbb{R}$. This equation is especially interesting in that when $m=\infty$, it is exactly the gradient Ricci soliton equation. When $\mathrm{f}$ is constant, it gives the Einstein equation and we call the quasi-Einstein metric trivial. When $m$ is a positive integer, it corresponds to warped product Einstein metrics.

Indeed, Case et al. [2011], elaborating on [Kim and Kim 2003], gave the following characterization of quasi-Einstein metrics.

Theorem 2. Let $M^{n} \times_{u} F^{m}$ be an Einstein warped product with Einstein constant $\lambda$, warping function $u=e^{-f / m}$ and Einstein fibre $F^{m}$. Then the weighted manifold ( $\left.M^{n}, g_{M}, e^{-f} d \mathrm{vol}\right)$ satisfies the quasi-Einstein equation (1). Furthermore the Einstein constant $\mu$ of the fibre satisfies

$$
\Delta f-|\nabla f|^{2}=m \lambda-m \mu e^{(2 / m) f} .
$$

Conversely if the weighted manifold $\left(M^{n}, g_{M}, e^{-f} d \mathrm{vol}\right)$ satisfies (1), then $f$ satisfies (2) for some constant $\mu \in \mathbb{R}$. Consider the warped product $N^{n+m}=M^{n} \times{ }_{u} F^{m}$ with $u=e^{-f / m}$, and Einstein fibre $F$ with ${ }^{F}$ Ric $=\mu g_{F}$. Then $N$ is Einstein with ${ }^{N}$ Ric $=\lambda g_{N}$.

\section{Scalar curvature estimates}

In this section, in the spirit of Theorem 3 of [Pigola et al. 2011], we generalize the scalar curvature estimates in Proposition 3.6 of [Case et al. 2011] to quasi-Einstein manifolds with non-constant scalar curvature. Possible rigidity at the endpoints is also discussed.

Theorem 3. Let $\left(M^{n}, g_{M}, e^{-f} d \mathrm{vol}\right)$ be a geodesically complete m-quasi-Einstein manifold, $1<m<+\infty$, with scalar curvature $S$, and let $S_{*}=\inf _{M} S$. 
(a) If $\lambda>0$, then $M$ is compact and

$$
\frac{n(n-1)}{m+n-1} \lambda<S_{*} \leq n \lambda .
$$

Moreover $S_{*} \neq n \lambda$ unless $M$ is Einstein.

(b) If $\lambda=0$ and $\inf _{M} f=f_{*}>-\infty$ then $S_{*}=0$. Moreover, either $S>0$ or $S(x) \equiv 0$. In the latter case, either $f$ is constant (and $M$ is trivial), or $M$ is isometric to the Riemannian product $\mathbb{R} \times \Sigma$, where $\Sigma$ is a Ricci-flat, totally geodesic hypersurface.

(c) If $\lambda<0$ and $\inf _{M} f=f_{*}>-\infty$, then

$$
n \lambda \leq S_{*} \leq \frac{n(n-1)}{m+n-1} \lambda
$$

and $S(x)>n \lambda$ unless $M$ is Einstein.

The proof of Theorem 3 will require the following formula obtained in [Case et al. 2011], which generalizes to the case $m<+\infty$ similar formulas for Ricci solitons $(m=+\infty)$ obtained previously by Petersen and Wylie [2009]. Following the terminology introduced in [Petersen and Wylie 2010], the $f$-Laplacian on the weighted manifold $\left(M, g_{M}, e^{-f} d \mathrm{vol}\right)$ is the diffusion-type operator defined by $\Delta_{f} u=e^{f} \operatorname{div}\left(\mathrm{e}^{-\mathrm{f}} \nabla \mathrm{u}\right)$. It is clearly a symmetric operator on $L^{2}\left(M, e^{-f} d \mathrm{vol}\right)$.

Lemma 4. Let $\operatorname{Ric}_{f}^{m}=\lambda g_{M}$, for some $\lambda \in \mathbb{R}$ and $m<+\infty$. Set $\tilde{f}=\frac{m+2}{m} f$. Then

$$
\frac{1}{2} \Delta_{\tilde{f}} S=-\frac{m-1}{m}\left|\operatorname{Ric}-\frac{1}{n} S g_{M}\right|^{2}-\frac{m+n-1}{m n}(S-n \lambda)\left(S-\frac{n(n-1)}{m+n-1} \lambda\right) .
$$

Proof of Theorem 3. First we show that $\inf _{M} S>-\infty$. According to Qian's version of Myers' theorem, this is obvious if $\lambda>0$ because $M$ is compact; see also the Appendix. In the general case, $\lambda \in \mathbb{R}$, we proceed as follows. Since

$$
-\left|\operatorname{Ric}-\frac{1}{n} S g_{M}\right|^{2}=-|\operatorname{Ric}|^{2}+\frac{S^{2}}{n},
$$

from (5) we obtain

$$
\begin{aligned}
\frac{1}{2} \Delta_{\tilde{f}} S & =-\frac{m-1}{m}|\operatorname{Ric}|^{2}-\frac{1}{m} S^{2}+\frac{m+2 n-2}{m} \lambda S-\frac{n(n-1)}{m} \lambda^{2} \\
& \leq-\frac{1}{m} S^{2}+\frac{m+2 n-2}{m} \lambda S .
\end{aligned}
$$

Let $S_{-}(x)=\max \{-S(x), 0\}$. Then

$$
\Delta_{\tilde{f}} S_{-} \geq \frac{2}{m} S_{-}^{2}+\frac{2(m+2 n-2)}{m} \lambda S_{-} .
$$

Now, from Qian's estimates of weighted volumes [1997] (see also [Mari et al. 2010, Section 2 and references]), since $\operatorname{vol}_{\tilde{f}}\left(B_{r}\right) \leq e^{-(2 / m) f_{*}} \operatorname{vol}_{f}\left(B_{r}\right)$, we can apply the a 
priori estimate in [Pigola et al. 2011, Theorem 12] to inequality (7) on the complete weighted manifold $\left(M, g_{M}, e^{-\tilde{f}} d \mathrm{vol}\right)$, and we obtain that $S_{-}$is bounded from above, or equivalently, $S_{*}=\inf _{M} S>-\infty$. Again from the volume estimates in [Qian 1997] and by Theorem 9 in [Pigola et al. 2011] applied to $\left(M, g_{M}, e^{-\tilde{f}} d \mathrm{vol}\right)$, the weak maximum principle at infinity for the $\tilde{f}$-Laplacian holds on $M$. This produces a sequence $\left\{x_{k}\right\}$, such that $\Delta_{\tilde{f}} S\left(x_{k}\right) \geq-1 / k$ and $S\left(x_{k}\right) \rightarrow S_{*}$. Taking the liminf in (5) along $\left\{x_{k}\right\}$ shows that, for $m>1$,

$$
0 \leq-\frac{m+n-1}{m n}\left(S_{*}-n \lambda\right)\left(S_{*}-\frac{n(n-1)}{m+n-1} \lambda\right) .
$$

We now distinguish three cases.

(a) Assume $\lambda>0$, so that $M$ is compact. Equation (8) yields

$$
\frac{n(n-1)}{m+n-1} \lambda \leq S_{*} \leq n \lambda \text {. }
$$

Assume now that $S_{*}=n \lambda>0$. Then $S \geq n \lambda \geq \frac{n(n-1)}{m+n-1} \lambda$, and from (5) we get

$$
\frac{1}{2} \Delta_{\tilde{f}} S \leq-\frac{m+n-1}{m n}(S-n \lambda)\left(S-\frac{n(n-1)}{m+n-1} \lambda\right) \leq 0 .
$$

Since $M$ is compact, $S$ must be constant. Hence, $S=S_{*}=n \lambda$. Substituting in (5), we obtain that Ric $=(1 / n) S g_{M}$, and thus that $M$ is Einstein.

Now we show that $S_{*}>(n(n-1) /(m+n-1)) \lambda$. Suppose that $S$ attains its minimum $(n(n-1) /(m+n-1)) \lambda$. Because the nonnegative function

$$
v(x)=S(x)-\frac{n(n-1)}{m+n-1} \lambda
$$

satisfies

$$
\frac{1}{2} \Delta_{\tilde{f}} v \leq-\frac{m+n-1}{m n} v^{2}+\lambda v \leq+\lambda v,
$$

and $v$ attains its minimum $v\left(x_{0}\right)=0$, it follows from the minimum principle [Gilbarg and Trudinger 1983, page 35] that $v$ vanishes identically. Hence,

$$
S \equiv \frac{n(n-1)}{m+n-1} \lambda
$$

is constant, and substituting in (5), we get that $M$ is Einstein with

$$
\text { Ric }=\frac{n-1}{m+n-1} \lambda g_{M} .
$$

Using this information with (1) we obtain that

$$
\operatorname{Hess}(f)=\frac{1}{m} d f \otimes d f+\frac{m}{m+n-1} \lambda g_{M}>0,
$$

but this is clearly impossible because $M$ is compact. 
(b) Assume $\lambda=0$. From (8) we conclude that $S_{*}=0$. Note that, according to (5), $\Delta_{\tilde{f}} S \leq 0$. Therefore, by the minimum principle, either $S(x)>0$ on $M$ or $S(x) \equiv 0$. In the latter case, substituting in (5), we obtain that $M$ is Ricci-flat and the $m$-quasi-Einstein equation reads $\operatorname{Hess}(f)-(1 / m) d f \otimes d f=0$. Therefore, either $f$ is constant and $M$ is Einstein, or the nonconstant function $u=e^{-f / m}$ satisfies Hess $(\mathrm{u})=0$. A Cheeger-Gromoll-type argument now shows that $M$ is isometric to the Riemannian product $\mathbb{R} \times \Sigma$ along the Ricci-flat, totally geodesic hypersurface $\Sigma$ of $M$.

(c) Assume $\lambda<0$. From (8) we deduce that $n \lambda \leq S_{*} \leq(n(n-1) /(m+n-1)) \lambda$. Suppose that $S\left(x_{0}\right)=n \lambda<0$ for some $x_{0} \in M$. Since the nonnegative function $w(x)=S(x)-n \lambda$ satisfies

$$
\frac{1}{2} \Delta_{\tilde{f}} w \leq-\frac{m+n-1}{m n} w^{2}-\lambda w \leq-\lambda w,
$$

and $w$ attains its minimum $w\left(x_{0}\right)=0$, it follows from the minimum principle that $w$ vanishes identically. Hence, $S \equiv n \lambda$ is constant, and substituting in (5) we get that $M$ is Einstein.

\section{Triviality results under $L^{p}$ conditions}

It is well known that steady or expanding compact Ricci solitons are necessarily trivial. The same result is proven in [Kim and Kim 2003] for quasi-Einstein metrics on compact manifolds with finite $m$. For Ricci solitons, a generalization to the complete, noncompact setting is obtained in [Pigola et al. 2011].

In this section, using the scalar curvature estimates of Theorem 3 , we get triviality for (not necessarily compact) quasi-Einstein metrics with $m<+\infty, \lambda \leq 0$.

Theorem 5. Let $\left(M^{n}, g_{M}, e^{-f} d \mathrm{vol}\right)$ be a geodesically complete noncompact $m$ quasi-Einstein manifold, $1<m<+\infty$. If the quasi-Einstein constant $\lambda$ is nonpositive and $f$ satisfies, for some $1<p<+\infty$,

$$
f \in L^{p}\left(M, e^{-f / m} d \mathrm{vol}\right),
$$

and $\inf _{M} f=f_{*}>-\infty$, then either $f \equiv \mathrm{const} \leq 0$ and $M$ is Einstein or $f>0$. Proof of Theorem 5. Tracing (1) and letting $\hat{f}=(1 / m) f$, we have

$$
\Delta_{\hat{f}} f=n \lambda-S .
$$

Since $\lambda \leq 0$ and $f_{*}>-\infty$, from (4) of Theorem 3 we obtain that $\Delta_{\hat{f}} f \leq 0$. Applying [Pigola et al. 2011, Theorem 14] to $f_{-}=\max \{-f, 0\} \in L^{p}\left(M, e^{-\hat{f}} d \mathrm{vol}\right)$, gives that $f_{-}$is constant. Hence, if there exists a point $x_{0} \in M$, such that $f\left(x_{0}\right) \leq 0$, then $f \equiv f\left(x_{0}\right) \leq 0$. 
Remark 6. From the proof, it follows that if either $M$ is compact or $f$ attains its absolute minimum, then $f \equiv$ const. Actually, it was pointed out to us by Dezhong Chen that the same conclusion holds if we merely assume that $f$ attains a local minimum at some point $x_{0} \in M$. The following proposition holds.

Proposition 7. Let $\left(M, g_{M}, e^{-f} d \mathrm{vol}\right)$ be a geodesically complete noncompact $m$ quasi-Einstein manifold, $1<m<+\infty$. If the quasi-Einstein constant $\lambda$ is nonpositive and $f$ satisfies $f_{*}>-\infty$, then any local minimum of $f$ is actually an absolute minimum.

Proof. Assume that $f$ attains a local minimum $x_{0} \in M$. Evaluating (10) at $x_{0}$, we get

$$
S\left(x_{0}\right) \leq n \lambda .
$$

Since $\lambda \leq 0$, by Theorem 3, $M$ is Einstein and $S$ is identically $n \lambda$. Thus the quasiEinstein equation (1) reads

$$
\operatorname{Hess}(f)=(1 / m) d f \otimes d f
$$

In particular, $\operatorname{Hess}(f)$ is positive semidefinite on $M$ and this implies the thesis.

\section{Proof of the main theorem}

Putting together the results of the previous sections, we easily obtain a proof of Theorem 1.

According to Theorem 2, $M$ is quasi-Einstein. Statement (a) follows immediately from Remark 6 and Proposition 7. In case (b), since $(n+m) \lambda={ }^{N} S \leq 0$, we get by Theorem 5 that $f$, and therefore $u$, is a constant function.

\section{Other triviality results}

Another triviality result for Einstein warped products was obtained by Case [2010]. Theorem 8 [Case 2010]. Let $N^{n+m}=M^{n} \times{ }_{u} F^{m}$ be a complete warped product with warping function $u(x)=e^{-f(x) / m}$, scalar curvature ${ }^{N} S \geq 0$ and complete Einstein fibre $F$. Then $N$ is simply a Riemannian product, provided the base manifold $M$ is complete, and the scalar curvature of $F$ satisfies ${ }^{F} S \leq 0$.

In the following theorem, we obtain the same conclusion in case the fibers have nonnegative scalar curvature, assuming an integrability condition on the warping function $u$. We observe that nontrivial examples with ${ }^{N} S \leq 0$ and ${ }^{F} S \geq 0$ are constructed in [Besse 1987, 9.118]. Thus, the integrabilty assumption is necessary.

Theorem 9. Let $N^{n+m}=M^{n} \times_{u} F^{m}$ be a complete Einstein warped product with warping function $u(x)=e^{-f(x) / m}$, scalar curvature ${ }^{N} S \leq 0$, and complete Einstein fibre $F$. Then $N$ is simply a Riemannian product, provided the base manifold $M$ 
is complete, the warping function satisfies $\int_{M} e^{-((p+m) / m) f} d \mathrm{vol}<+\infty$ for some $1<p<+\infty$, and the scalar curvature of $F$ satisfies ${ }^{F} S \geq 0$. In this case $M$ and $F$ are Ricci-flat and $M$ is compact.

Combining Theorem 8 and Theorem 9 immediately gives the following.

Corollary 10. Let $N$ be a complete Ricci-flat warped product with complete Einstein fibre $F$ and warping function $u(x)=e^{-f(x) / m}$ satisfying $u \in L^{p}\left(M, e^{-f} d \mathrm{vol}\right)$, for some $1<p<+\infty$. Then $N$ is simply a Riemannian product.

Proof of Theorem 9. Just observe that computing the $f$-Laplacian of $u$ and using (2), one obtains

$$
\Delta_{f} u=\mu u^{-1}-\lambda u+\frac{u}{m^{2}}|\nabla f|^{2} .
$$

Thus, in our assumptions, we obtain that $\Delta_{f} u \geq 0$. Since $0<u \in L^{p}\left(M, e^{-f} d \mathrm{vol}\right)$ [Pigola et al. 2011, Theorem 14], we obtain the constancy of $u$. Up to a rescaling of the metric of $F$, we can suppose $u=1$.

Now, since the Riemannian product $M \times F$ is Einstein, both $M$ and $F$ are Einstein manifolds with the same Einstein constant. In particular, ${ }^{M} S$ and ${ }^{F} S$ have the same sign. By our assumption on the signs of ${ }^{N} S$ and ${ }^{F} S$, we thus obtain that both $M$ and $F$ are Ricci-flat. Finally, since $u$ (and thus $f$ ) is constant, from the integrability condition, we obtain $\operatorname{vol}(M)<+\infty$. Thus, by a result of Calabi and Yau (see [Yau 1976]), we obtain that $M$ is compact.

We end this section with a nonexistence result. Recall that by the volume estimates in [Qian 1997] and by [Pigola et al. 2011, Theorem 9], the weak maximum principle for the $f$-Laplacian holds on $\left(M, g_{M}, e^{-f} d \mathrm{vol}\right)$, provided $\mathrm{Ric}_{f}^{m}=\lambda g_{M}$ for some $\lambda \in \mathbb{R}, m<+\infty$.

Theorem 11. There is no complete Einstein warped product $N=M^{n} \times{ }_{u} F^{m}$ with warping function $u=e^{-f / m} \in L^{\infty}(M)$, scalar curvature ${ }^{N} S<0$ and Einstein fibre $F$ with ${ }^{F} S \geq 0$.

Proof. Since $m \mu={ }^{F} S \geq 0$, from (12) we have

$$
\Delta_{f} u \geq-\lambda u .
$$

Since, by assumption, $u$ satisfies $\sup _{M} u=u^{*}<+\infty$, by the weak maximum principle at infinity for the $f$-Laplacian, there exists a sequence $\left\{x_{k}\right\} \subset M$, along which $u\left(x_{k}\right) \geq u^{*}-1 / k$ and $\Delta_{f} u\left(x_{k}\right) \leq 1 / k$. Thus evaluating (13) along $\left\{x_{k}\right\}$ and taking the limit as $k \rightarrow+\infty$, we obtain that $\lambda u^{*} \geq 0$, and since $u^{*}>0$, we cannot have $\lambda<0$. 


\section{Appendix}

An extension of Myers' theorem to weighted manifolds with a positive lower bound on the $m$-Bakry-Emery Ricci tensor ( $m$ finite) was obtained by Qian [1997]. For generalizations of Myers' theorem in a different direction, see [Morgan 2006].

In this section we extend Qian's theorem by allowing some negativity of the $m$ Bakry-Emery Ricci tensor. Our considerations begin with the following Bochner formula for the $m$-Bakry-Emery Ricci tensor; see, for example, [Setti 1998].

Let $u: M^{n} \rightarrow \mathbb{R}$ be a smooth function on a complete weighted manifold

$$
\left(M^{n}, g_{M}, e^{-f} d \mathrm{vol}\right) \text {. }
$$

Then

$$
\begin{aligned}
& \frac{1}{2} \Delta_{f}|\nabla u|^{2} \\
& \quad=|\operatorname{Hess}(u)|^{2}+g_{M}\left(\nabla u, \nabla \Delta_{f} u\right)+\operatorname{Ric}_{f}^{m}(\nabla u, \nabla u)+\frac{1}{m}\left|g_{M}(\nabla f, \nabla u)\right|^{2} .
\end{aligned}
$$

With this formula one obtains the following generalization of a well-known lemma, which estimates the integral of Ricci along minimizing geodesics. The proof is modeled on [Qian 1997].

Lemma 12. Let $\left(M^{n}, g_{M}, e^{-f} d \mathrm{vol}\right)$ be a complete weighted manifold, and consider the $m$-Bakry-Emery Ricci tensor Ric ${ }_{f}^{m}$ for $m$ finite. Fix $o \in M$ and let $r(x)=\operatorname{dist}(x, o)$. For any point $q \in M$, let $\gamma_{q}:[0, r(q)] \rightarrow M$ be a minimizing geodesic from o to $q$, such that $\left|\dot{\gamma}_{q}\right|=1$. If $h \in \operatorname{Lip}_{\text {loc }}(\mathbb{R})$ is such that $h(0)=h(r(q))=0$, then for every $q \in M$, it holds that

$$
0 \leq \int_{0}^{r(q)}(m+n-1)\left(h^{\prime}\right)^{2} d s-\int_{0}^{r(q)} h^{2} \operatorname{Ric}_{f}^{m}\left(\dot{\gamma}_{q}, \dot{\gamma}_{q}\right) d s .
$$

Proof. Fix a point $q \notin \operatorname{cut}(o)$. Straightforward computations show that

$$
\begin{aligned}
\frac{\left(\Delta_{f} r\right)^{2}}{m+n-1} & \leq \frac{(\Delta r)^{2}}{n-1}+\frac{\left|g_{M}(\nabla f, \nabla r)\right|^{2}}{m}, \\
|\operatorname{Hess}(r)|^{2} & \geq \frac{(\Delta r)^{2}}{n-1} .
\end{aligned}
$$

Using (16) and (17), from the Bochner formula (14) applied to the distance function $r(x)$, we obtain that

$$
0 \geq \frac{\left(\Delta_{f} r\right)^{2}}{m+n-1}+g_{M}\left(\nabla r, \nabla \Delta_{f} r\right)+\operatorname{Ric}_{f}^{m}(\nabla r, \nabla r) .
$$

Evaluating this along a minimizing geodesic $\gamma_{q}$, such that $\left|\dot{\gamma}_{q}\right|=1$, we get

$$
0 \geq \frac{\left(\Delta_{f} r \circ \gamma_{q}\right)^{2}}{m+n-1}+\frac{d}{d s}\left(\Delta_{f}\left(r \circ \gamma_{q}\right)\right)+\operatorname{Ric}_{f}^{m}\left(\dot{\gamma}_{q}, \dot{\gamma}_{q}\right) .
$$


If $h \in \operatorname{Lip}_{\text {loc }}(\mathbb{R}), h \geq 0$, and $h(0)=0$, then multiplying (18) by $h^{2}$ and integrating on $[0, t]$, we obtain

$$
0 \geq \int_{0}^{t} h^{2} \frac{\left(\Delta_{f} r \circ \gamma_{q}\right)^{2}}{m+n-1} d s+\int_{0}^{t} \frac{d}{d s}\left(\Delta_{f} r \circ \gamma_{q}\right) h^{2} d s+\int_{0}^{t} h^{2} \operatorname{Ric}_{f}^{m}\left(\dot{\gamma}_{q}, \dot{\gamma}_{q}\right) d s .
$$

Since $\left(\Delta_{f} r \circ \gamma_{q}\right) h^{2} \rightarrow 0$ as $r \rightarrow 0$, integrating by parts, we have

$$
\begin{aligned}
0 \geq \int_{0}^{t} h^{2} \frac{\left(\Delta_{f} r \circ \gamma_{q}\right)^{2}}{m+n-1} d s & +h^{2}(t)\left(\Delta_{f} r \circ \gamma_{q}\right)(t) \\
& -2 \int_{0}^{t} h h^{\prime}\left(\Delta_{f} r \circ \gamma_{q}\right) d s+\int_{0}^{t} h^{2} \operatorname{Ric}_{f}^{m}\left(\dot{\gamma}_{q}, \dot{\gamma}_{q}\right) d s .
\end{aligned}
$$

Since

$$
-2 h h^{\prime}\left(\Delta_{f} r \circ \gamma_{q}\right) \geq \frac{-h^{2}\left(\Delta_{f} r \circ \gamma_{q}\right)^{2}}{m+n-1}-(m+n-1)\left(h^{\prime}\right)^{2},
$$

we deduce that

$$
0 \geq h^{2}(t)\left(\Delta_{f} r \circ \gamma_{q}\right)-\int_{0}^{t}(m+n-1)\left(h^{\prime}\right)^{2} d s+\int_{0}^{t} \operatorname{Ric}_{f}^{m}\left(\dot{\gamma}_{q}, \dot{\gamma}_{q}\right) h^{2} d s .
$$

Thus, taking $t=r(q)$ and choosing $h$ such that $h^{2}(r(q))=0$, we get (15) for $q \notin \operatorname{cut}(o)$. To treat the general case, one can use the Calabi trick. Namely, suppose that $q \in \operatorname{cut}(o)$. Translating the origin $o$ to $o_{\epsilon}=\gamma_{q}(\epsilon)$ so that $q \notin \operatorname{cut}\left(o_{\epsilon}\right)$, using the triangle inequality, and finally, taking the limit as $\epsilon \rightarrow 0$, one checks that (15) also holds in this case.

From Lemma 12, some Myers-type results can be proven. Here we generalize a theorem of Galloway [1979].

Theorem 13. Let $\left(M^{n}, g_{M}, e^{-f} d \mathrm{vol}\right)$ be a complete weighted manifold. Given two different points $p, q \in M$, let $\gamma_{p, q}$ be a minimizing geodesic from $p$ to $q$ parameterized by arc length. Suppose that there exist constants $c$ and $G \geq 0$ such that for each pair of points $p, q$, it holds that

$$
\left.\operatorname{Ric}_{f}^{m}\left(\dot{\gamma}_{p, q}, \dot{\gamma}_{p, q}\right)\right|_{\gamma_{p, q}(t)} \geq(m+n-1)\left[c^{2}+\frac{d}{d t}\left(g \circ \gamma_{p, q}\right)\right],
$$

for some $C^{1}(M)$ function $g$ satisfing $\sup _{M}|g| \leq G, m<+\infty$. Then $M$ is compact and

$$
\operatorname{diam}(M) \leq \frac{1}{c}\left[\frac{2 G}{c}+\sqrt{\frac{4 G^{2}}{c^{2}}+\pi^{2}}\right] .
$$

Proof. Define $L$ to be the length of $\gamma_{p, q}$ between $p$ and $q$ and set

$$
h(t):=\sin \left(\frac{\pi}{L} t\right) .
$$




\section{Compute}

$\int_{0}^{L} h^{2}(t) d t=\int_{0}^{L} \sin ^{2}\left(\frac{\pi}{L} t\right) d t=\frac{L}{2}, \quad \int_{0}^{L} h^{\prime 2}(t) d t=\frac{\pi^{2}}{L^{2}} \int_{0}^{L} \cos ^{2}\left(\frac{\pi}{L} t\right) d t=\frac{\pi^{2}}{2 L}$.

Then, applying Lemma 12, we have

$$
\begin{aligned}
& \frac{\pi^{2}(m+n-1)}{2 L} \\
& \quad=\int_{0}^{L}(m+n-1) h^{\prime 2} \geq\left.\int_{0}^{L} h^{2} \operatorname{Ric}_{f}^{m}\left(\dot{\gamma}_{p, q}, \dot{\gamma}_{p, q}\right)\right|_{\gamma_{p, q}} d s \\
& \geq c^{2}(m+n-1) \int_{0}^{L} h^{2}+(m+n-1) \int_{0}^{L} h^{2} \frac{d}{d t}\left(g \circ \gamma_{p, q}\right) \\
& \quad=\frac{c^{2}(m+n-1) L}{2}+\left.(m+n-1) h^{2} g\left(\gamma_{p, q}\right)\right|_{0} ^{L} \\
& \quad-(m+n-1)\left[\int_{0}^{L / 2}\left(\frac{d}{d t} h^{2}\right)\left(g \circ \gamma_{p, q}\right)+\int_{L / 2}^{L}\left(\frac{d}{d t} h^{2}\right)\left(g \circ \gamma_{p, q}\right)\right] \\
& \geq \frac{c^{2}(m+n-1) L}{2}-(m+n-1) G\left[\int_{0}^{L / 2}\left(\frac{d}{d t} h^{2}\right)+\int_{L / 2}^{L}\left|\frac{d}{d t} h^{2}\right|\right] \\
& \geq \frac{c^{2}(m+n-1) L}{2}-2(m+n-1) G .
\end{aligned}
$$

Finally, this can be written as

$$
c^{2} L^{2}-4 G L-\pi^{2} \leq 0,
$$

which in turn implies (20), because $p$ and $q$ are arbitrary.

Reasoning as in the classical case [Galloway 1982; Mastrolia et al. 2011], the validity of (15) and an integration by parts show that the compactness of $M$ depends on the behavior, and on the position of the zeros, of the solution of the differential equation along minimizing geodesics

$$
-h^{\prime \prime}(t)-\frac{\operatorname{Ric}_{f}^{m}(\dot{\gamma}, \dot{\gamma})}{m+n-1} h(t)=0 .
$$

It remains to find sufficient conditions on $\mathrm{Ric}_{f}^{m}$ for which solutions of the differential equation (22) have a first zero at finite time. Minor changes to the proofs of the results contained in [Mastrolia et al. 2011] lead to similar compactness results in the weighted setting. In particular, we state the following theorem, in which a Myers-type conclusion is obtained assuming a nonpositive lower bound on $\mathrm{Ric}_{f}^{m}$.

Theorem 14. Let $\operatorname{Ric}_{f}^{m} \geq-(m+n-1) B^{2}$, for some constant $B \geq 0, m<+\infty$. Suppose there is a point $q \in M$ such that along each geodesic $\gamma:[0,+\infty) \rightarrow M$ 
parameterized by arc length, with $\gamma(0)=q$, it holds that either

$$
\int_{a}^{b} t \frac{\operatorname{Ric}_{f}^{m}(\dot{\gamma}, \dot{\gamma})}{m+n-1} d t>B\left\{b+a \frac{e^{2 B a}+1}{e^{2 B a}-1}\right\}+\frac{1}{4} \log \left(\frac{b}{a}\right),
$$

or

$$
\int_{a}^{b} t^{\alpha} \frac{\operatorname{Ric}_{f}^{m}(\dot{\gamma}, \dot{\gamma})}{m+n-1}(t) d t>B\left\{b^{\alpha}+a^{\alpha} \frac{e^{2 B a}+1}{e^{2 B a}-1}\right\}+\frac{\alpha^{2}}{4(1-\alpha)}\left\{a^{\alpha-1}-b^{\alpha-1}\right\}
$$

for some $0<a<b$ and $\alpha \neq 1$. Then $M$ is compact.

\section{Acknowledgement}

The author is deeply grateful to his advisor Stefano Pigola for his guidance and constant encouragement during the preparation of the manuscript. The author would also like to thank Dezhong Chen for pointing out to him Proposition 7.

\section{References}

[Besse 1987] A. L. Besse, Einstein manifolds, Ergebnisse der Math. und ihrer Grenzgebiete (3) 10, Springer, Berlin, 1987. MR 88f:53087 Zbl 0613.53001

[Case 2010] J. S. Case, “The nonexistence of quasi-Einstein metrics”, Pacific J. Math. 248:2 (2010), 277-284. MR 2741248 Zbl 1204.53032

[Case et al. 2011] J. Case, Y.-J. Shu, and G. Wei, "Rigidity of quasi-Einstein metrics", Differential Geom. Appl. 29:1 (2011), 93-100. MR 2784291 Zbl 1215.53033

[Galloway 1979] G. J. Galloway, “A generalization of Myers' theorem and an application to relativistic cosmology", J. Differential Geom. 14:1 (1979), 105-116 (1980). MR 81i:53049 Zbl 0444. 53036

[Galloway 1982] G. J. Galloway, "Compactness criteria for Riemannian manifolds", Proc. Amer. Math. Soc. 84:1 (1982), 106-110. MR 83a:53040 Zbl 0484.53032

[Gilbarg and Trudinger 1983] D. Gilbarg and N. S. Trudinger, Elliptic partial differential equations of second order, 2nd ed., Grundlehren der Mathematischen Wissenschaften 224, Springer, Berlin, 1983. MR 86c:35035 Zbl 0562.35001

[Kim and Kim 2003] D.-S. Kim and Y. H. Kim, "Compact Einstein warped product spaces with nonpositive scalar curvature", Proc. Amer. Math. Soc. 131:8 (2003), 2573-2576. MR 2004b:53063 Zbl 1029.53027

[Mari et al. 2010] L. Mari, M. Rigoli, and A. G. Setti, "Keller-Osserman conditions for diffusiontype operators on Riemannian manifolds", J. Funct. Anal. 258:2 (2010), 665-712. MR 2011c:58041 Zbl 1186.53051

[Mastrolia et al. 2011] P. Mastrolia, M. Rimoldi, and G. Veronelli, "Myers' type theorems and some related oscillation results", J. Geom. Anal. (2011). arXiv 1002.2076

[Morgan 2006] F. Morgan, "Myers' theorem with density", Kodai Math. J. 29:3 (2006), 455-461. MR 2007h:53043 Zbl 1132.53306

[Petersen and Wylie 2009] P. Petersen and W. Wylie, "Rigidity of gradient Ricci solitons", Pacific J. Math. 241:2 (2009), 329-345. MR 2010j:53071 Zbl 1176.53048 
[Petersen and Wylie 2010] P. Petersen and W. Wylie, "On the classification of gradient Ricci solitons”, Geom. Topol. 14:4 (2010), 2277-2300. MR 2740647 Zbl 1202.53049

[Pigola et al. 2011] S. Pigola, M. Rimoldi, and A. Setti, "Remarks on non-compact gradient Ricci solitons", Math. Z. 268:3-4 (2011), 777-790. arXiv 0905.2868v3

[Qian 1997] Z. Qian, "Estimates for weighted volumes and applications", Quart. J. Math. Oxford Ser. (2) 48:190 (1997), 235-242. MR 98e:53058 Zbl 0902.53032

[Setti 1998] A. G. Setti, "Eigenvalue estimates for the weighted Laplacian on a Riemannian manifold”, Rend. Sem. Mat. Univ. Padova 100 (1998), 27-55. MR 2000a:58082 Zbl 0922.58084

[Yau 1976] S. T. Yau, "Some function-theoretic properties of complete Riemannian manifold and their applications to geometry", Indiana Univ. Math. J. 25:7 (1976), 659-670. MR 54 \#5502 Zbl 0335.53041

Received July 8, 2010.

MiCHELE RIMOLDI

Dipartimento Di MATEMATICA F. ENRIQUES

UNIVERSITÀ DEGLI STUdI DI MILANO

I-20133 MILAN

ITALY

michele.rimoldi@unimi.it 


\title{
PACIFIC JOURNAL OF MATHEMATICS
}

\author{
http://www.pjmath.org \\ Founded in 1951 by
}

E. F. Beckenbach (1906-1982) and F. Wolf (1904-1989)

\section{EDITORS}

V. S. Varadarajan (Managing Editor)

Department of Mathematics

University of California

Los Angeles, CA 90095-1555

pacific@math.ucla.edu

Vyjayanthi Chari

Department of Mathematics

University of California

Riverside, CA 92521-0135

chari@math.ucr.edu

\section{Robert Finn}

Department of Mathematics Stanford University

Stanford, CA 94305-2125

finn@math.stanford.edu

Kefeng Liu

Department of Mathematics

University of California

Los Angeles, CA 90095-1555

liu@math.ucla.edu
Darren Long

Department of Mathematics

University of California

Santa Barbara, CA 93106-3080

long@math.ucsb.edu

Jiang-Hua Lu

Department of Mathematics

The University of Hong Kong

Pokfulam Rd., Hong Kong jhlu@maths.hku.hk

Alexander Merkurjev

Department of Mathematics University of California

Los Angeles, CA 90095-1555 merkurev@math.ucla.edu
Sorin Popa

Department of Mathematics

University of California

Los Angeles, CA 90095-1555

popa@math.ucla.edu

Jie Qing

Department of Mathematics

University of California

Santa Cruz, CA 95064

qing@ cats.ucsc.edu

Jonathan Rogawski

Department of Mathematics

University of California

Los Angeles, CA 90095-1555

jonr@math.ucla.edu

\section{PRODUCTION}

pacific@math.berkeley.edu

Silvio Levy, Scientific Editor Matthew Cargo, Senior Production Editor

ACADEMIA SINICA, TAIPEI

CALIFORNIA INST. OF TECHNOLOGY

INST. DE MATEMÁTICA PURA E APLICADA

KEIO UNIVERSITY

MATH. SCIENCES RESEARCH INSTITUTE

NEW MEXICO STATE UNIV.

OREGON STATE UNIV.

\section{SUPPORTING INSTITUTIONS}

STANFORD UNIVERSITY
UNIV. OF BRITISH COLUMBIA
UNIV. OF CALIFORNIA, BERKELEY
UNIV. OF CALIFORNIA, DAVIS
UNIV. OF CALIFORNIA, LOS ANGELES
UNIV. OF CALIFORNIA, RIVERSIDE
UNIV. OF CALIFORNIA, SAN DIEGO
UNIV. OF CALIF., SANTA BARBARA

UNIV. OF CALIF., SANTA CRUZ

UNIV. OF MONTANA

UNIV. OF OREGON

UNIV. OF SOUTHERN CALIFORNIA

UNIV. OF UTAH

UNIV. OF WASHINGTON

WASHINGTON STATE UNIVERSITY

These supporting institutions contribute to the cost of publication of this Journal, but they are not owners or publishers and have no responsibility for its contents or policies.

See inside back cover or www.pjmath.org for submission instructions.

The subscription price for 2011 is US \$420/year for the electronic version, and \$485/year for print and electronic.

Subscriptions, requests for back issues from the last three years and changes of subscribers address should be sent to Pacific Journal of Mathematics, P.O. Box 4163, Berkeley, CA 94704-0163, U.S.A. Prior back issues are obtainable from Periodicals Service Company, 11 Main Street, Germantown, NY 12526-5635. The Pacific Journal of Mathematics is indexed by Mathematical Reviews, Zentralblatt MATH, PASCAL CNRS Index, Referativnyi Zhurnal, Current Mathematical Publications and the Science Citation Index.

The Pacific Journal of Mathematics (ISSN 0030-8730) at the University of California, c/o Department of Mathematics, 969 Evans Hall, Berkeley, CA 94720-3840, is published monthly except July and August. Periodical rate postage paid at Berkeley, CA 94704, and additional mailing offices. POSTMASTER: send address changes to Pacific Journal of Mathematics, P.O. Box 4163, Berkeley, CA 94704-0163.

PJM peer review and production are managed by EditFLOW ${ }^{\mathrm{TM}}$ from Mathematical Sciences Publishers.

PUBLISHED BY PACIFIC JOURNAL OF MATHEMATICS

at the University of California, Berkeley 94720-3840

A NON-PROFIT CORPORATION

Typeset in IATEX

Copyright $(2011$ by Pacific Journal of Mathematics 


\section{PACIFIC JOURNAL OF MATHEMATICS}

Volume $252 \quad$ No. $1 \quad$ July 2011

Some dynamic Wirtinger-type inequalities and their applications

RaVi P. Agarwal, Martin Bohner, Donal O'REgAn and SAMIR H.

SAKER

Splitting criteria for vector bundles on higher-dimensional varieties

PARSA BAKHTARY

Average Mahler's measure and $L_{p}$ norms of unimodular polynomials

KWOK-KWOng STEPHen CHOI and Michael J. MossinghofF

Tate resolutions and Weyman complexes

David A. CoX and EVgeny Materov

On pointed Hopf algebras over dihedral groups

FERNANDO FANTINO and GASTON ANDRÉS GARCIA

Integral topological quantum field theory for a one-holed torus

PATRicK M. Gilmer and Gregor Masbaum

Knot 4-genus and the rank of classes in $\boldsymbol{W}(\mathbb{Q}(t))$

CHARLES LIVINGSTON

Roots of Toeplitz operators on the Bergman space

ISSAM LOUHICHI and NAGISETTY V. RAO

Uniqueness of the foliation of constant mean curvature spheres in asymptotically 145 flat 3-manifolds

SHIGUANG MA

On the multiplicity of non-iterated periodic billiard trajectories

MARCo MAZZUCCHELLI

A remark on Einstein warped products

MiCHELE RIMOLDI

Exceptional Dehn surgery on large arborescent knots

YING-QING WU

Harnack estimates for the linear heat equation under the Ricci flow 